\title{
Osteomielitis por Yokenella regensburgei después de una craneotomía en una paciente inmunocompetente
}

\author{
Sara Catalina Penagos ${ }^{1}$, Sebastián Gómez ${ }^{1}$, Pablo Villa², Santiago Estrada ${ }^{3}$, Carlos Andrés Agudelo² \\ 1 Facultad de Medicina, Universidad Pontificia Bolivariana, Medellín, Colombia \\ 2 Clínica Universitaria Bolivariana, Medellín, Colombia \\ ${ }^{3}$ Laboratorio Clínico VID, Congregación Mariana, Medellín, Colombia
}

\begin{abstract}
Yokenella regensburgei es un bacilo Gram negativo de la familia Enterobacteriaceae, que puede encontrarse en agua de pozos, alimentos y en el tubo digestivo de insectos y reptiles. Aunque se ha aislado de muestras provenientes de seres humanos, pocas veces se ha reportado como causante de infección y, en tales casos, especialmente en pacientes inmunosuprimidos. Se presenta aquí el primer caso de osteomielitis secundaria a una infección por $Y$. regensburgei en una paciente inmunocompetente después de un procedimiento quirúrgico.
\end{abstract}

Palabras clave: Enterobacteriaceae, osteomielitis.

doi: http://dx.doi.org/10.7705/biomedica.v35i4.2719

\begin{abstract}
Osteomyelitis due to Yokenella regensburgei following craniotomy in an immunocompetent patient
The gram-negative bacillus Yokenella regensburgei (of the Enterobacteriaceae family) can be found in groundwater and foodstuffs, as well as the digestive tracts of insects and reptiles. Although it has been isolated from humans since its original description, it has rarely been reported as a cause of infection, and then, only in immunosuppressed patients. We report the first case of post-surgical secondary osteomyelitis due to $Y$. regensburgei in an immunocompetent woman who had undergone a craniotomy.
\end{abstract}

Key words: Enterobacteriaceae, osteomyelitis.

doi: http://dx.doi.org/10.7705/biomedica.v35i4.2719

Yokenella regensburgei es un bacilo Gram negativo de la familia Enterobacteriaceae, que puede encontrarse en agua de pozos, en alimentos y en el tubo digestivo de insectos y reptiles (1-3). Aunque se ha aislado de muestras provenientes de seres humanos, pocas veces se ha reportado como causante de infección, por lo que la información sobre la epidemiología, los mecanismos de transmisión y el comportamiento clínico, es limitada (1). Por las semejanzas bioquímicas con Hafnia alvei, que confunden a los sistemas automatizados de identificación, probablemente su incidencia se ha subestimado (4).

\footnotetext{
Correspondencia:

Pablo Villa, Clínica Universitaria, Universidad Pontificia Bolivariana, Calle 78B N69-240, Medellín, Colombia

Teléfono: (574) 445 5900, extensión 795

pablovillaf@hotmail.com

Recibido: 02/07/14; aceptado: 14/05/15
}

La mayoría de los casos reportados de infección por $Y$. regensburgei en seres humanos (seis hasta enero de 2014 registrados en Medline), se ha presentado en individuos con algún grado de inmunosupresión, y su presentación clínica ha sido variable. Se presenta aquí el primer caso de osteomielitis causada por este organismo en una mujer inmunocompetente, después de la resección de un macroadenoma hipofisiario.

\section{Descripción del caso}

Se trata de una paciente de 70 años de edad sin antecedentes patológicos de importancia, residente en el área rural del departamento de Antioquia, Colombia, que fue sometida a la resección de un macroadenoma hipofisario invasor, productor de prolactina, que afectaba la región selar y se extendía al espacio subaracnoideo comprometiendo el quiasma y el tracto óptico izquierdo, lo que le había producido síntomas visuales. El Grupo de Neurocirugía del hospital se

\footnotetext{
Contribución de los autores:

Todos los autores participaron en la búsqueda bibliográfica y en la redacción del artículo final.
} 
encargó de la resección transcraneana del tumor, sin que se presentara ninguna complicación en el periodo posoperatorio inmediato. Seis semanas después de la cirugía, la paciente presentó edema y eritema en la zona quirúrgica, que progresaron a pesar del tratamiento inicial con cefazolina $y$ clindamicina en el hospital local, por lo que fue remitida a nuestra institución.

En el examen físico de ingreso, la paciente presentaba eritema, calor, secreción purulenta en la herida quirúrgica y ptosis palpebral ipsilateral, sin compromiso inflamatorio periorbitario. Los resultados de los exámenes de laboratorio iniciales revelaron un recuento de leucocitos normal $(6.610$ células $/ \mathrm{mm}^{3}$, neutrófilos: $46 \%$ ). La proteína C reactiva, la velocidad de sedimentación globular, la función renal y los electrolitos, fueron normales. En la tomografía computadorizada simple y la contrastada, se observó una colección líquida subdural laminar y captación meníngea del medio de contraste a nivel del parietal izquierdo, así como una pequeña colección líquida en los tejidos blandos epicraneanos, de 25 por $8 \mathrm{~mm}$, en la región frontal izquierda y atenuación heterogénea sin reacción perióstica en la tabla ósea del área de la craneotomía (figura 1).

Se hizo una craneotomía, se drenó la colección líquida (100 ml en la región epidural y $10 \mathrm{ml}$ en la subdural) y se tomaron muestras para los cultivos. Tres días después del procedimiento quirúrgico, se obtuvo el crecimiento de bacilos Gram negativos en el cultivo de hueso y de las colecciones alojadas en las zonas epidural y subdural, que posteriormente se tipificaron como $Y$. regensburgei. El aislamiento se confirmó utilizando el sistema de identificación Vitek $^{\circledR} 2$ compact y se corroboró con el sistema Phoenix ${ }^{\circledR}$, con un intervalo de confianza del $99 \%$ en ambos casos. Con base en el perfil de sensibilidad (cuadro 1), se cambió el tratamiento antibiótico a $400 \mathrm{mg}$ de ciprofloxacina cada ocho horas por vía intravenosa; dada la evolución favorable de la paciente, se decidió completar 42 días de tratamiento con $750 \mathrm{mg}$ cada 12 horas por vía oral. Un año después, no se había presentado recurrencia de la infección.

\section{Discusión}

Yokenella regensburgei es un bacilo Gram negativo de la familia Enterobacteriaceae, identificado inicialmente como del biogrupo 9 en el Instituto Nacional de Salud del Japón. Posteriormente, en los Centers for Disease Control and Prevention (CDC) de Atlanta se le denominó Koserella trabulsii y se le clasificó dentro del grupo entérico 45, aunque finalmente se mantuvo el nombre de Yokonella regensburgei dado por quienes primero lo describieron $(1,5)$. Su nombre se deriva de la abreviación en japonés del Instituto Nacional de Salud de Japón (Yoken) y del diminutivo de "ella" en latín. El nombre de la especie, regensburgei, se le dio en honor a la región de Regnesburg, Alemania, en donde se aisló por primera vez de un insecto $(1,5,6)$.
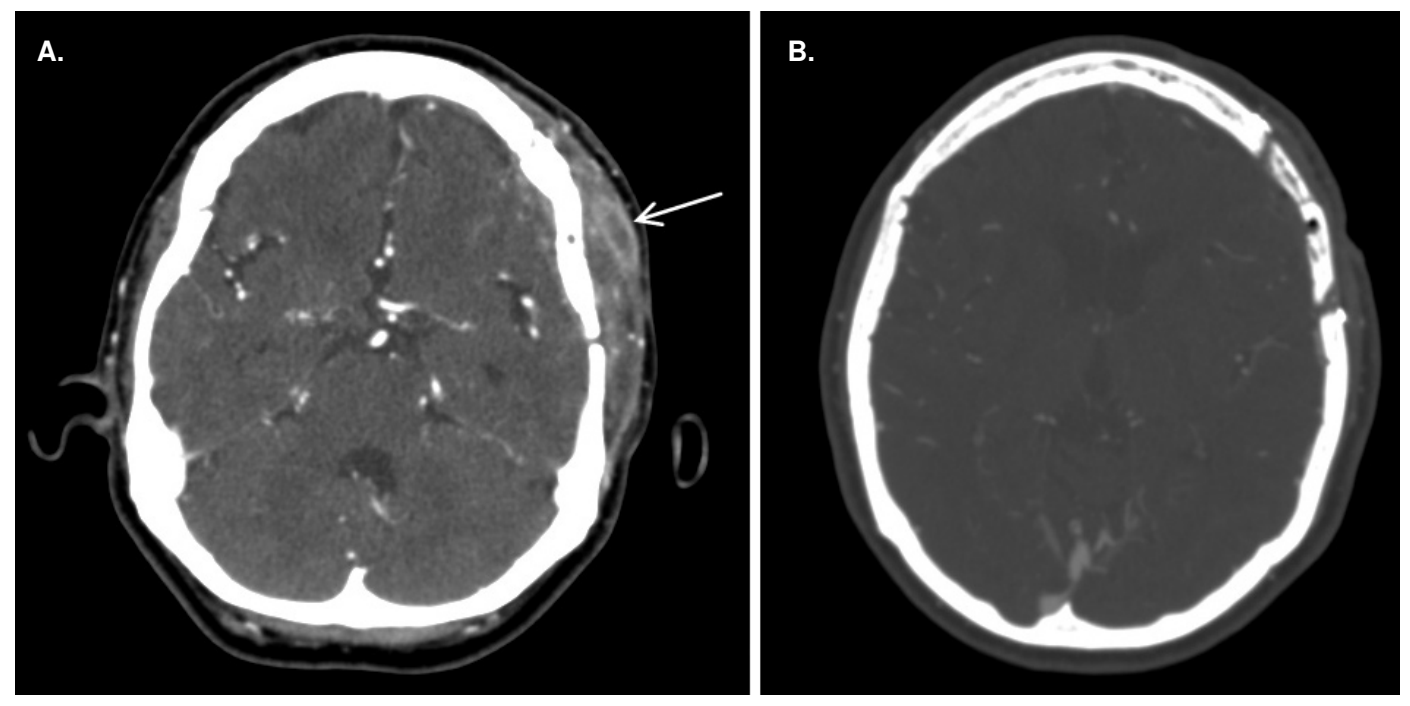

Figura 1. Corte axial de tomografía computadorizada craneal

A. Engrosamiento notable de los tejidos blandos en la región parietal izquierda y una pequeña colección líquida asociada (flecha).

B. Aspecto después de la cirugía en el hueso temporal, con un patrón erosivo secundario a cambios inflamatorios e infecciosos. 
Cuadro 1. Perfil de sensibilidad de Yokonella regensburgei

\begin{tabular}{lcc}
\hline Antibiótico & Sensibilidad & $\begin{array}{c}\text { Concentración } \\
\text { inhibitoria mínima }\end{array}$ \\
\hline Amikacina & Sensible & $\leq 2$ \\
Cefepime & Sensible & $\leq 1$ \\
Ciprofloxacina & Sensible & $\leq 0,25$ \\
Ertapenem & Sensible & $\leq 0,5$ \\
Gentamicina & Sensible & $\leq 1$ \\
Meropenem & Sensible & $\leq 0,25$ \\
\hline
\end{tabular}

El bacilo rara vez se ha reportado como causa de infección en seres humanos, por lo que poco se sabe de su patogenia, características bioquímicas, epidemiología y perfil de sensibilidad a los diferentes antibióticos (7). La mayoría de los pacientes reportados hasta el momento, presentaba algún tipo de inmunosupresión (abuso de alcohol, uso crónico de esteroides, insuficiencia renal, trasplante de órgano sólido o de precursores hematopoyéticos y diabetes mellitus, entre otros), por lo que se ha supuesto que $Y$. regensburgei es un patógeno oportunista (7-11). A diferencia de los casos anteriores, esta paciente no presentaba ningún grado de inmunosupresión al momento de la infección. Otro caso conocido de infección en un paciente inmunocompetente se reportó en un niño indio de cinco años con bacteriemia por $Y$. regensburgei sin causa identificada (12).

Yokonella regensburgei no se ha reportado como causa de infecciones en el sitio operatorio, ni como causa de aquellas asociadas a la atención en salud. Con la información disponible, es imposible establecer si el germen se adquirió durante el procedimiento quirúrgico o si la infección de la herida ocurrió después del alta hospitalaria. Este tipo de enterobacteria se ha aislado en muestras de aguas de pozos y, al menos en uno de los casos reportados, la persona había estado expuesta a agua contaminada durante una inundación masiva, por lo que se sugirió una posible asociación entre la infección y el uso de agua no tratada y almacenada en tanques $(5,11)$.

En la mayoría de los casos reportados, las infecciones producidas por $Y$. regensburgei han comprometido tejidos blandos, aunque también se ha encontrado en infecciones de las vías urinarias, en bacteriemias y en artritis, así como en muestras provenientes de las vías respiratorias y del tubo digestivo de humanos (1,4,5,7-12). En el caso que se describe, se aisló en muestras de hueso y de la colección líquida en tejidos blandos en las zonas epidural y subdural, las cuales se tomaron mediante procedimiento quirúrgico bajo condiciones estériles, lo que hace poco probable una contaminación. Este es el primer caso conocido de osteomielitis por Y. regensburgei.

Las pruebas de identificación automatizadas son muy confiables hoy en día, pues permiten diferenciar claramente entre los organismos de la familia Enterobacteriaceae, especialmente $H$. alvei, el cual propició las clasificaciones erróneas hechas en un comienzo. Yokonella regensburgei es negativa en la reacción de Voges-Proskauer, malonato y glicerol, y positiva en la reacción de citrato y melibiosa. Por el contrario, $H$. alvei es positiva en la reacción de Voges-Proskauer, malonato y glicerol, y negativa en la reacción de citrato y melibiosa $(13,14)$.

No se ha establecido el antibiótico de elección para el tratamiento de las infecciones por $Y$. regensburgei y existen pocos datos sobre su sensibilidad a los diferentes antimicrobianos. En un estudio de 10 cepas de Y. regensburgei, Stock, et al., documentaron la presencia de genes $a m p C$ y la expresión de potentes betalactamasas muy inducibles estrechamente relacionadas con las enzimas AmpC cromosómicas de Enterobacter spp. y Citrobacter spp. En este mismo estudio se documentó muy buena actividad de los carbapenémicos con concentraciones inhibitorias mínimas inferiores a $0,25 \mathrm{mg} / \mathrm{L}$ en todas las cepas estudiadas, y de las quinolonas, con concentraciones inferiores a 0,03 $\mathrm{mg} / \mathrm{L}$, lo que confirma su gran sensibilidad a los dos antimicrobianos administrados a esta paciente y explica el buen resultado con el tratamiento. Otros grupos de antibióticos a los cuales $Y$. regensburgei es sensible, incluyen los aminoglucósidos, las tetraciclinas, el cloramfenicol, los inhibidores de la síntesis del ácido fólico y la fosfomicina (4).

En resumen, se reporta el primer caso de osteomielitis del cráneo por $Y$. regensburgei en una paciente inmunocompetente, con muy buen resultado terapéutico con quinolonas.

\section{Conflicto de intereses}

Los autores declaran no tener conflictos de intereses respecto a la investigación, autoría y publicación de este artículo.

\section{Financiación}

No se contó con financiación para la elaboración de este reporte de caso. 


\section{Referencias}

1. Kosaco Y, Sakazaki R, Yoshizaki E. Yokenella regensburgei gen. nov., sp. nov.: A new genus and species in the family Enterobacteriaceae. Jpn J Med Sci Biol. 1984;37:117-24.

2. Perira-de Oliveira SM, de Morais BA, Gonçalves CA, Giordano-Dias CM, Vilela ML, Brazil RP, et al. Digestive tract microbiota in female Lutzomyia longipalpis (Lutz \& Neiva, 1912) (Diptera: Psychodidae) from colonies feeding on blood meal and sucrose plus blood meal. Cad Saúde Pública. 2001;17:229-32. http://dx.doi.org/10.1590/S0102$311 X 2001000100024$

3. Cassel-Béraud AM, Richard C. The aerobic intestinal flora of the microchiropteran bat Chaerephon pumila in Madagascar. Bull Soc Pathol Exot Filiales.1988;81: 806-10.

4. Stock I, Sherwood KJ, Wiedemann B. Antimicrobial susceptibility patterns, $\beta$-lactamases, and biochemical identification of Yokenella regensburgei strains. Diag Microbiol Infect Dis. 2004;48:5-15. http://dx.doi.org/10. 1016/j.diagmicrobio.2003.08.006

5. Hickman-Brenner FW, Huntley-Carter GP, Fanning GR, Brenner DJ, Farmer JJ 3rd. Koserella trabulsii, a new genus and species of Enterobacteriaceae formerly known as Enteric Group 45. J Clin Microbiol. 1985;21:39-42.

6. Brenner DJ, Krieg NR, Staley JT. Bergey's Manual of Systematic Bacteriology. Second edition. East Lansing, MI: Springer US; 2005. p. 587-607.
7. Bhowmick T, Weinstein MP. A deceptive case of cellulitis caused by a gram-negative pathogen. J Clin Microbiol. 2013;51:1320-3. http://dx.doi.org/10.1128/JCM.02975-12

8. Abbott SL, Janda JM. Isolation of Yokenella regensburgei ("Koserella trabulsii") from a patient with transient bacteremia and from a patient with a septic knee. J Clin Microbiol. 1994;32:2854-55.

9. Fajardo-Olivares M, Blanco-Palenciano J, MárquezLaffon I, Ruiz-León JM. Yokenellaregensburgei infection in a perimalleolar ulcer. Med Clin (Barc). 2005;125:358-9.

10. Semler MW, Richter JA, Petri WA. Yokenella regensburgei as a cause of sepsis in an immunocompromised patient. University of Virginia Journal of Medicine. 2008;2:19-21.

11. Lo YC, Chuang YW, Lin YH. Yokenella regensburgei in an immunocompromised host: A case report and review of the literature. Infection. 2011;39:485-8. http://dx.doi. org/10.1007/s15010-011-0139-z

12. Jain S, Gaind R, Gupta KB, Dawar R, Kumar D, Paul P, et al. Yokenella regensburgei infection in India mimicking enteric fever. J Med Microbiol. 2013;62:935-9. http://dx.doi. org/10.1099/jmm.0.058669-0

13. Winn W, Allen SD, Janda WM, Koneman EW, Procop GW, Schrenckenberger PC, et al. Diagnóstico microbiológico. Texto y atlas en color. Sexta edición. Buenos Aires: Editorial Panamericana; 2008. p. 205-88.

14. Versalovik J, Carroll KC, Funke G, Jorgensen JH, Landry ML, Warnock DW. Manual of Clinical Microbiology. Tenth edition. Washington, D.C.: ASM Press; 2011. p. 639-57. 